\title{
Computationally Feasible VCG Mechanisms
}

\author{
Noam Nisan \\ School of Computer Science and Engineering, \\ The Hebrew University of Jerusalem, Israel
}

\author{
Amir Ronen \\ Faculty of Industrial Engineering 83 Management, \\ Technion - Israel Institute of Technology, \\ Haifa 32000, Israel
}

NOAM@CS.HUJI.AC.IL

AMIRR@IE.TECHNION.AC.IL

\begin{abstract}
A major achievement of mechanism design theory is a general method for the construction of truthful mechanisms called VCG (Vickrey, Clarke, Groves). When applying this method to complex problems such as combinatorial auctions, a difficulty arises: VCG mechanisms are required to compute optimal outcomes and are, therefore, computationally infeasible. However, if the optimal outcome is replaced by the results of a sub-optimal algorithm, the resulting mechanism (termed VCG-based) is no longer necessarily truthful. The first part of this paper studies this phenomenon in depth and shows that it is near universal. Specifically, we prove that essentially all reasonable approximations or heuristics for combinatorial auctions as well as a wide class of cost minimization problems yield non-truthful VCG-based mechanisms. We generalize these results for affine maximizers.

The second part of this paper proposes a general method for circumventing the above problem. We introduce a modification of VCG-based mechanisms in which the agents are given a chance to improve the output of the underlying algorithm. When the agents behave truthfully, the welfare obtained by the mechanism is at least as good as the one obtained by the algorithm's output. We provide a strong rationale for truth-telling behavior. Our method satisfies individual rationality as well.
\end{abstract}

\section{Introduction}

Mechanism design is a sub-field of game theory and microeconomics that studies the design of protocols for non-cooperative environments. In such environments the participating agents follow their own goals and do not necessarily act as instructed by the mechanism. This theory has traditionally been applied to economic applications such as auctions of various kinds. An introduction to mechanism design can be found in several books (Osborne \& Rubinstein, 1994; Mas-Collel, Whinston, \& Green, 1995). In recent years, problems on the border of mechanism design and computer science have attracted the attention of many researchers, both within and outside the AI community. In particular, mechanism design models were applied to multi-agent systems (Rosenschein \& Zlotkin, 1994; Wellman, Wurman, Walsh, \& MacKie-Mason, 2001; Shoham \& Tanaka, 1997; Shoham \& Tennenholtz, 2001), decentralized resource and task allocations (Nisan \& Ronen, 2001; Wellman et al., 2001; Elkind, Sahai, \& Steiglitz, 2004; Porter, Ronen, Shoham, \& Tennenholtz, 2002), economic and electronic commerce applications (Parkes, 1999; Cramton, 1997), and communication networks (Feigenbaum, Papadimitriou, \& Shenker, 2000; Anderson, Kelly, \& Steinberg, 2002). 
The canonical mechanism design problem can be described as follows: A set of rational agents needs to collaboratively choose an outcome $o$ from a finite set $O$ of possibilities. Each agent $i$ has a privately known valuation function $v^{i}: O \rightarrow R$ quantifying the agent's benefit from each possible outcome. The agents are supposed to report their valuation functions $v^{i}(\cdot)$ to some centralized mechanism. The goal of the mechanism is to choose an outcome $o$ that maximizes the total welfare $\sum_{i} v^{i}(o)$. The main difficulty is that agents may choose to misreport their valuations in an attempt to affect the outcome to their liking. Such manipulations are likely to severely damage the resulting welfare (simulations that demonstrate this welfare loss can be found in Carroll \& Grosu, 2005). The tool that the mechanism uses to motivate the agents to reveal the truth is monetary payments. These payments need to be designed in a way that ensures that rational agents always reveal their true valuations. Mechanisms with this property are called incentive compatible or truthful (in dominant strategies). To date, only one general method, called VCG (Vickrey, 1961; Clarke, 1971; Groves, 1973) (or slightly more generally, affine maximization), is known for designing such a payment structure ${ }^{1}$. In some settings, it is known that this method is the sole available one (Roberts, 1979; Lavi, Nisan, \& Mualem, 2003).

Many novel applications of mechanism design are complex and require implementation on computer systems. Cases in point include combinatorial auctions where multiple items are concurrently sold in an auction (Cramton, Shoham, \& Steinberg, 2006), decentralized task and resource allocation problems (Nisan \& Ronen, 2001; Wellman et al., 2001), and networking applications (Feigenbaum et al., 2000; Anderson et al., 2002). For many of these applications, the range of possible outcomes is huge and even finding an outcome that maximizes the total welfare is known to be NP-complete. Since for such cases computing the optimal outcome is intractable, the VCG method cannot be applied.

A natural general approach for the development of mechanisms for such cases would be to use a sub-optimal polynomial time algorithm for computing the outcome, and calculate the payments by applying the VCG payment rule to the underlying algorithm. We term such mechanisms VCG-based.

The starting point of this paper is the observation, noted already by some researchers (Lehmann, O`Callaghan, \& Shoham, 2002; Nisan \& Ronen, 2001), that VCG-based mechanisms are not necessarily truthful. Thus, rational agents may lie, taking advantage of quirks in the outcome determination algorithm.

\subsection{VCG-based Mechanisms are Generally not Truthful}

The first part of this paper examines this last phenomenon in depth and shows that it is near universal: essentially all reasonable VCG-based mechanisms are not truthful.

We first turn our attention to combinatorial auctions and characterize the class of truthful VCG-based mechanisms for this problem ${ }^{2}$. We say that an allocation algorithm for

1. Recently, a few truthful mechanisms, which are not affine maximizers, were obtained for combinatorial auctions (Bartal, Gonen, \& Nisan, 2003).

2. The importance of combinatorial auctions is twofold. First, they have direct applications such as FCC auctions. Second, they abstract many problems of resource allocation among self-interested agents. A comprehensive survey of research on combinatorial auctions can be found in a recent book (Cramton et al., 2006). 
combinatorial auctions is reasonable if, whenever an item is desired by a single agent only, this agent receives the item. The above characterization leads to the following corollary:

Theorem: Any truthful VCG-based mechanism for combinatorial auctions is not reasonable (unless it uses the exponential optimal allocation algorithm).

In particular, unless $P=N P$, every polynomial time, truthful VCG-based mechanism is not reasonable.

Loosely speaking, we show that essentially the only degree of freedom available to truthful VCG-based mechanisms is the choice of range over which to optimize. Within this range perfect optimization is needed. This theorem seems intuitive as VCG payments identify each agent's utility with that of society, and thus if the social welfare is not optimized by the mechanism, then agents are motivated to to lie in order to do so. Yet, such an argument only shows that the outcome must be locally optimal - with locality defined as a deviation by a single agent. The heart of our argument is a delicate hybrid argument showing that in a general context this local optimization essentially implies global optimization.

Next we study a family of problems termed cost minimization allocation problems. This family contains many natural decentralized task allocation problems such as mechanism design versions of the shortest path problem (Elkind et al., 2004; Nisan \& Ronen, 2001; Rosenschein \& Zlotkin, 1994). We call a mechanism for such a problem degenerate if there exist inputs that cause it to produce results that are arbitrarily far from the optimal.

Theorem: For any cost minimization allocation problem, any sub-optimal truthful VCGbased mechanism is degenerate.

A word is in order here about the significance of these results. VCG-based mechanisms are not just some special case of truthful mechanisms - they are essentially the only general method known for truthful mechanisms in non-single dimensional settings. Moreover, in certain settings they are known to indeed be the only truthful mechanisms (Roberts, 1979; Lavi et al., 2003). More precisely, weighted versions of VCG-based mechanisms - called affine maximizers - are truthful, but our results extend (as we show) to these cases as well. Consequently, our results imply that designing truthful mechanisms for computationally intractable problems requires either restricting the range of the outcomes (getting "unreasonable" or "degenerate" mechanisms) or developing entirely new techniques for truthful mechanisms - which may not even exist. A similar implication results if the intractability stems not from computational considerations, but rather from communication considerations (Cramton et al., 2006, Chapter 11).

\subsection{The Second Chance Mechanism}

The second part of this paper proposes a general method for circumventing the difficulty of constructing truthful mechanisms. While VCG-based mechanisms lose their incentive compatibility, they still pose a very special property. Loosely speaking, in such a mechanism, the only reason for an agent to misreport its valuation is to "help" the algorithm compute a better outcome. We would like to exploit this property to obtain mechanisms that are "almost" truthful. 
Given any algorithm for the corresponding optimization problem we define the second chance mechanism based on it. This mechanism is a modification of the VCG-based mechanism where, in addition to their valuations, the agents are allowed to submit appeal functions. An appeal function allows the agent to give the algorithm an input (a vector of declared valuations), which is different from the original input, but without misreporting its type. When the agents behave truthfully, the welfare obtained by the mechanism is at least as good as the one obtained by the algorithm's output.

We then formulate the rationale for truthful behavior in our mechanism. Informally, our argument is as follows: Under reasonable assumptions, in any situation in which the agent believes it is beneficial for it to lie to the mechanism, it is better for the agent to report its actual type to the mechanism and ask its appeal to check whether this lie really helps it. Thus, the agent can construct a truthful strategy premised on the fact that it is not aware of any situation in which another strategy would be better. We believe that this is a strong argument for truth-telling.

We construct a version of our mechanism that satisfies individual rationality as well. A generalization of our results to affine maximization and to compensation and bonus mechanisms (Nisan \& Ronen, 2001) is straightforward.

Several alternative approaches aimed at handling the difficulty of developing truthful mechanisms were suggested in the past. One approach is the construction of mechanisms that are computationally hard to manipulate (e.g., Bartholdi et al., 1992). To the best of our knowledge such manipulations are only hard in the worst case (e.g., it may be NPhard to always compute such a manipulation). Nevertheless, such hardness does not rule out the possibility that manipulations may be easy to compute in "typical" cases. Another possible approach is to consider other equilibria of VCG (Holzman, Kfir-Dahav, Monderer, \& Tennenholtz, 2004; Holzman \& Monderer, 2004). However, there is no apparent way for the agents to coordinate such equilibria. Several recent works construct ascending mechanisms for combinatorial auctions (e.g. Parkes, 1999). Such mechanisms rely on assumptions about

the agents which are very different from ours (e.g., myopic behavior). It may be interesting to compare the virtues of such mechanisms to those of ours.

A few multi-round mechanisms for combinatorial auctions that let the agents improve the provisional allocation were proposed and tested in the past (Banks, Ledyard, \& Porter, 1989). Our argument for truthfulness in second chance mechanisms may provide a partial explanation for the relative success reported in these experiments.

\section{Preliminaries}

In this section we formally present our model. We attempt as much as possible to use the standard notions of both mechanism design and computational complexity theories.

\subsection{Mechanism Design Problems}

This section formulates the class of mechanism design problems that we study.

Definition 1 (utilitarian mechanism design problem) $A$ (utilitarian) mechanism design problem is described by:

1. A finite set $O$ of allowed outputs. 
2. Each agent $i=(1, \ldots, n)$ has a real function $v^{i}(o \in O)$ called its valuation or type. This is a quantification of its benefit from each possible output o in terms of some common currency. $v^{i}($.$) is privately known to agent i$.

3. If the mechanism's output is o and in addition the mechanism hands the agent $p^{i}$ units of currency, then its utility $u^{i}$ equals ${ }^{3} v^{i}(o)+p^{i}$. This utility is what the agent aims to optimize.

4. The goal of the mechanism is to select an output $o \in O$ that maximizes the total welfare $g(v, o)=\sum_{i} v^{i}(o)$.

An example of such a problem can be found in Section 2.4.

Note that the goal in these problems is to maximize the total welfare but not necessarily the revenue. This goal, also known as economic efficiency, is justified in many settings and is extensively studied in economics.

In a direct revelation mechanism, the participants are simply asked to reveal their types to the mechanism. Based on these declarations the mechanism computes the output $o$ and the payment $p^{i}$ for each of the agents.

Definition 2 (mechanism) $A$ (direct revelation) mechanism is a pair $m=(k, p)$ such that:

- The output function $k$ accepts as input a vector $w=\left(w^{1}, \ldots, w^{n}\right)$ of declared valuation functions $^{4}$ and returns an output $k(w) \in O$.

- The payment function $p(w)=\left(p^{1}(w), \ldots, p^{n}(w)\right)$ returns a real vector quantifying the payment handed by the mechanism to each of the agents (e.g. if $p^{i}=2$, the mechanism pays two units of currency to agent $i$ ).

The agents try to maximize their own utility and thus may lie to the mechanism. As these lies might severely reduce the total welfare, the mechanism should be carefully designed such that it will be for the benefit of the agents to report their types truthfully.

Notation: We denote the tuple $\left(a^{1}, \ldots a^{i-1}, a^{i+1}, \ldots, a^{n}\right)$ by $a^{-i}$. We let $\left(a^{i}, a^{-i}\right)$ denote the tuple $\left(a^{1}, \ldots, a^{n}\right)$.

Definition 3 (truthful mechanism) A mechanism is called truthful if truth-telling is a dominant strategy, i.e., for every agent $i$ of type $v^{i}$ and for every type declaration $w^{-i}$ for the other agents, the agent's utility is maximized when it declares its real valuation function $v^{i}$.

As an example consider the famous Vickrey auction (Vickrey, 1961): A seller wishes to sell one item in an auction. There are $n$ buyers, each privately knowing its valuation $v^{i}$ for this item. (The value for not winning is assumed to be zero.) In a Vickrey auction each of the buyers is simply asked for its valuation; the item is allocated to the buyer with the

3. This assumption is called quasi-linearity and is very common in mechanism design.

4. We do not consider the issue of how to represent the valuations. 
highest bid for the price of the second highest. The reader may verify that this mechanism is truthful. Another example of a truthful mechanism can be found in Section 2.4.

In general, the communication protocol of a mechanism can be complicated. A simple observation known as the revelation principle for dominant strategies (e.g., Mas-Collel et al., 1995, pp. 871) states that for every mechanism where the agents have dominant strategies, there exists an equivalent truthful mechanism. Thus, w.l.o.g. it is possible to focus on truthful mechanisms.

\subsection{VCG-based Mechanisms}

This subsection presents the celebrated VCG mechanisms. Intuitively, these mechanisms solve utilitarian problems by identifying the utility of truthful agents with the declared total welfare. We then generalize these mechanisms.

Definition 4 (VCG mechanism, (via Groves, 1973)) A mechanism $m=(k, p)$ belongs to the VCG family if:

- $k(w)$ maximizes the total welfare according to $w$. That is, for all $w, k(w) \in \arg \max _{o} g(w, o)$.

- The payment is calculated according to the VCG formula: $p^{i}(w)=\sum_{j \neq i} w^{j}(k(w))+$ $h^{i}\left(w^{-i}\right)\left(h^{i}(\right.$.$\left.) is an arbitrary function of w^{-i}\right)$.

The reader may verify that the Vickrey auction is a VCG mechanism. It is well known that VCG mechanisms are truthful (Groves, 1973).

Unfortunately, for many applications, the task of finding an output $k(w)$ that maximizes the total welfare is computationally infeasible (e.g., NP-hard). In this paper we consider VCG mechanisms where the optimal algorithm is replaced by a sub-optimal but computationally feasible one.

Definition 5 (VCG-based mechanism) Let $k(w)$ be an algorithm that maps type declarations into allowable outputs. We call $m=(k(w), p(w))$ a VCG mechanism based on $k($.$) if p($.$) is calculated according to the VCG formula: p^{i}(w)=\sum_{j \neq i} w^{j}(k(w))+h^{i}\left(w^{-i}\right)$ (where $h^{i}($.$) is an arbitrary function of w^{-i}$ ).

Obviously, a VCG-based mechanism that is based on an optimal algorithm is a VCG mechanism. Note that the payment function of a VCG-based mechanism is not identical to the VCG payment because the algorithm $k($.$) is plugged into the payment formula. We will now$ characterize the utility of an agent in VCG-based mechanisms. This utility is equivalent to the total welfare according to the declared types of the other agents and the actual type of the agent under consideration.

Lemma 2.1 (VCG-based utility) Consider a VCG-based mechanism defined by the allocation algorithm $k($.$) , and the functions \left(h^{1}(),. \ldots, h^{n}().\right)$. Suppose that the actual valuation of agent $i$ is $v^{i}$, and the declarations are $w=\left(w^{1}(),. \ldots, w^{n}().\right)$. Then the utility of agent $i$ equals $g\left(\left(v^{i}, w^{-i}\right), k(w)\right)+h^{i}\left(w^{-i}\right)$. 
Proof: The proof is immediate from the definitions. The agent's utility equals $v^{i}(k(w))+$ $p^{i}(w)=v^{i}(k(w))+\sum_{j \neq i} v^{j}(k(w))+h^{i}\left(w^{-i}\right)=g\left(\left(v^{i}, w^{-i}\right), k(w)\right)+h^{i}\left(w^{-i}\right)$.

In other words, a VCG-based mechanism identifies the utility of truthful agents with the total welfare. In particular, when $k($.$) is optimal, g\left(\left(v^{i}, w^{-i}\right), k(w)\right)$ is maximized when the agent is truthful. This implies that VCG mechanisms are truthful but this truthfulness is not necessarily preserved by VCG-based mechanisms.

\subsubsection{Example: Non Optimal Vickrey Auction}

This section demonstrates the problems that might occur when the optimal algorithm in a VCG mechanism is replaced by a sub-optimal one. Consider the sale of a single item. As we already commented, a Vickrey auction is a VCG mechanism. Its algorithm allocates the item to the agent with the highest declared value. The function $h^{i}\left(w^{-i}\right)=-\sum_{j \neq i} w^{j}(o)$ equals the negation of the second highest value in case $i$ is winning.

Consider the same mechanism where the optimal algorithm is replaced by an algorithm that only chooses the second highest agent. The mechanism will now give the object to the agent with the second highest declaration for the price of the third highest agent.

Suppose that there are three agents. Alice who has a value of $\$ 2$ million, Bob with a value of $\$ 1.7$ million, and Carol who has a value of $\$ 1$ million. When the agents are truthful, Bob wins and pays $\$ 1$ million. In this case it is for Alice's benefit to reduce her declaration below Bob's. Similarly, if Alice wins, Bob would like to lower his declaration further, and so on. Note that there are natural situations where Carol will win as well.

It is not difficult to see that there are no dominant strategies in this game. The outcome of the mechanism is highly unpredictable, depending heavily on the agents' beliefs about the others, their risk attitude, and their level of sophistication. Such a mechanism can yield inefficient outcomes. The efficiency loss may get much worse when the underlying optimization problem has a complex combinatorial structure (simulations that demonstrate this in the context of scheduling were done by Carroll \& Grosu, 2005).

\subsubsection{AfFine-BASED MECHANisms}

It is possible to slightly generalize the class of VCG mechanisms and obtain mechanisms called affine maximizers. Such mechanisms maximize affine transformations of the valuations. When the domain of valuations is unrestricted, affine maximizers are the sole truthful mechanisms (Roberts, 1979; Lavi et al., 2003). Similarly to VCG, we generalize these mechanisms to incorporate sub-optimal algorithms.

Notation: Let $a=\left(a_{0}, \ldots, a_{n}\right)$ be an $n+1$-tuple such that $a_{0}($.$) is a valuation function,$ and $a_{1}, \ldots, a_{n}$ are strictly positive. We define the weighted welfare $g_{a}(w, o)$ of an output $o$ as $a_{0}(o)+\sum_{i>0} a_{i} \cdot w_{i}(o)$ where $w$ is a vector of types and $o$ an output.

Definition 6 (affine-based mechanism) Let $k(w)$ be an algorithm that maps type declarations into allowable outputs, $a=\left(a_{0}, \ldots, a_{n}\right)$ be an $n+1$-tuple such that $a_{0}($.$) is a$ valuation function, and $a_{1}, \ldots, a_{n}$ are strictly positive. We call $m=(k(w), p(w))$ an affine mechanism based on $k$ if $p$ is calculated according to the formula: $p^{i}(w)=\frac{1}{a_{i}}\left(\sum_{j \neq i, 0} a_{j}\right.$. $\left.w^{j}(k(w))+h^{i}\left(w^{-i}\right)\right)$ (where $h^{i}()$ is an arbitrary function of $\left.w^{-i}\right)$. 
The function $a_{0}($.$) can be interpreted as the preferences of the mechanism over the set of$ the alternatives and $a_{1}, \ldots, a_{n}$ as weights over the agents. As in VCG mechanisms, the agents' utility have a convenient characterization.

Lemma 2.2 (affine-based utility) Consider an affine-based mechanism defined by the allocation algorithm $k($.$) , a tuple a$ and the functions $h^{1}(),. \ldots, h^{n}($.$) . Suppose that the$ actual valuation of agent $i$ is $v^{i}$, and the declarations are $w=\left(w^{1}(),. \ldots, w^{n}().\right)$. Then the utility of agent $i$ equals $\frac{1}{a_{i}}\left(g_{a}\left(\left(v^{i}, w^{-i}\right), k(w)\right)+h^{i}\left(w^{-i}\right)\right)$.

Proof: The proof is immediate from the definitions. The agent's utility equals $v^{i}(k(w)+$ $\left.p^{i}(w)\right)=\frac{1}{a_{i}}\left(a_{i} v^{i}(k(w))+p^{i}(w)=\frac{1}{a_{i}}\left(g_{a}\left(\left(v^{i}, w^{-i}\right), k(w)\right)+h^{i}\left(w^{-i}\right)\right)\right.$.

In other words, an affine-based mechanism identifies the agents' utility with the affine transformation of the valuations it aims to optimize. In particular, when $k($.$) maximizes$ $g_{a}(w,$.$) , the mechanism is truthful.$

\subsection{Computational Considerations in Mechanism Design}

This section adopts standard notions of computational complexity to revelation mechanisms.

Definition 7 (polynomial mechanism) A mechanism $(k, p)$ is called polynomial time computable if both $k(w)$ and $p(w)$ run in polynomial time (in the size of $w$ ).

Note that a VCG-based mechanism is polynomial iff its output algorithm and the functions $h^{i}($.$) are polynomial. We sometimes call polynomial algorithms and mechanisms$ computationally feasible.

Definition 8 (NP-complete problem) A mechanism design problem is called NP-Complete if the problem of finding an output that maximizes the total welfare is NP-Complete.

We use the term feasible to denote "acceptable" computational time and infeasible for unacceptable computational time. In particular, NP-hard problems and exponential algorithms are considers infeasible, while polynomial algorithms are considered feasible. We use these non-standard terms because most of our results are not limited to specific complexity classes.

\subsection{Example: Combinatorial Auctions}

The problem of combinatorial auctions has been extensively studied in recent years (a recent book can be found at Cramton et al., 2006). The importance of this problem is twofold. Firstly, several important applications rely on it (e.g., the FCC auction Cramton, 1997). Secondly, it is a generalization of many other problems of interest, in particular in the field of electronic commerce.

The problem: A seller wishes to sell a set $S$ of items (radio spectra licenses, electronic devices, etc.) to a group of agents who desire them. Each agent $i$ has, for every subset $s \subseteq S$ of the items, a non-negative number $v^{i}(s)$ that represents how much $s$ is worth for it. $v^{i}($.$) is privately known to each agent i$. We make two standard additional assumptions on the agents' type space: 
No externalities The valuation of each agent $i$ depends only on the items allocated to it. In other words, for every two allocations $x=\left(x_{1}, \ldots, x_{n}\right)$ and $y=\left(y_{1}, \ldots, y_{n}\right)$, if $x_{i}=y_{i}$, then $v^{i}(x)=v^{i}(y)$. Thus, we denote the valuation of each agent $i$ by $v^{i}: 2^{S} \rightarrow R$.

Free disposal Items have non-negative values, i.e., if $s \subseteq t$, then $v^{i}(s) \leq v^{i}(t)$. Also, $v^{i}(\phi)=0$.

Items can either be complementary, i.e., $v^{i}(S \bigcup T) \geq v^{i}(S)+v^{i}(T)$, or substitutes, i.e., $v^{i}(S \bigcup T) \leq v^{i}(S)+v^{i}(T)$ (for disjoint $S$ and $T$ ). For example, a buyer may be willing to pay $\$ 200$ for a T.V. set, $\$ 150$ for a VCR, $\$ 450$ for both and only $\$ 200$ for two VCRs.

If agent $i$ gets the set $s^{i}$ of items, and its payment is $p^{i}$, its utility is $v^{i}\left(s^{i}\right)+p^{i}$. (The payments in combinatorial auctions are non-positive.) This utility is what each agent tries to optimize. For example, an agent prefers to buy a $\$ 1000$ valued VCR for $\$ 600$, gaining $\$ 400$, rather buy a $\$ 1500$ valued VCR for $\$ 1250$.

In a VCG mechanism for a combinatorial auction, the participants are first required to reveal their valuation functions to the mechanism. The mechanism then computes, according to the agents' declarations, an allocation $s$ that maximizes the total welfare. The payment for each of the agents is calculated according to the VCG formula. By Lemma 2.1, the utility $u^{i}=v^{i}\left(s^{i}\right)+p^{i}$ of each of the agents is maximized when it reveals its true valuation to the mechanism. When all agents are truthful, the mechanism maximizes the total welfare.

Consider, however, the computational task faced by such a mechanism. After the types are declared, the mechanism needs to select, among all possible allocations, one that maximizes the total welfare. This problem is known to be NP-Complete. Therefore, unless the number of agents and items is small, such a mechanism is computationally infeasible. Even the problem of finding an allocation that approximates the optimal allocation within

a reasonable factor of $\sqrt{|S|}-\epsilon$ is $N P$-Complete (Zuckerman, 2006). Nevertheless, various heuristics and tractable sub-cases have been analyzed in the literature (Cramton et al., 2006, Chapter 13). We would like to find a way to turn these sub-optimal algorithms into mechanisms.

We note that, in general, revealing a valuation function requires exponential communication. While we ignore communication issues in this paper, a subsequent work (Ronen, 2001) extends the second chance method to address communication limitations as well.

\section{Limitations of Truthful VCG-based Mechanisms}

This section studies the limitations of truthful VCG-based mechanisms. Section 3.1 characterizes these mechanisms for the important problem of combinatorial auctions (see Section 2.4). This characterization precludes the possibility of obtaining truthfulness by applying VCG rules to many of the proposed heuristics for combinatorial auctions (e.g., the greedy algorithms of Lehmann et al., 2002 and Nisan, 2000). Moreover, we show that any truthful non-optimal VCG-based mechanism for combinatorial auctions suffers from abnormal behavior. Section 3.2 shows that for many natural cost minimization problems, any truthful VCG-based mechanism is either optimal or produces results that are arbitrarily far from 
optimal. As a result, when such a problem is computationally intractable, any truthful computationally feasible VCG-based mechanism has inputs that cause it to produce degenerate results. Furthermore, since standard algorithmic techniques do not yield such anomalies, it might be difficult to develop algorithms that can be plugged into truthful mechanisms. We generalize these results to affine-based mechanisms as well.

\subsection{Truthful VCG-based Mechanisms for Combinatorial Auctions}

This section characterizes the class of truthful VCG-based mechanisms for combinatorial auctions.

Definition 9 (maximal in its range) Let $k(w)$ be an algorithm that maps type declarations into allowable outputs. Let $V \stackrel{d f}{=} \prod_{i=1}^{n} V^{i}$ be the space of all possible types and let $V^{\prime} \subseteq V$ be a subspace of $V$. Let $\mathcal{O}$ denote the range of $k$ at $V^{\prime}$, i.e. $\mathcal{O}=\left\{k(w) \mid w \in V^{\prime}\right\}$. We say that $k$ is maximal in its range at $V^{\prime}$ if for every type $w \in V^{\prime}, k(w)$ maximizes $g$ over $\mathcal{O}$. We say that $k$ is maximal in its range if it is maximal in its range at $V$.

Consider, for example, an algorithm for combinatorial auctions that allocates all the items (the set $S$ ) to the agent with the highest valuation $v^{i}(S)$. Clearly, this polynomial time algorithm is maximal in its range. The welfare obtained by the allocation of this algorithm achieves at least a factor of $\max (1 / n, 1 /|S|)$ of the optimal welfare (where $n$ denotes the number of agents).

Proposition 3.1 A VCG-based mechanism with an output algorithm that is maximal in its range is truthful.

Proof: Such a mechanism is a VCG mechanism where the set of allowable outputs is the range of its output algorithm. By Lemma 2.1 such a mechanism is truthful.

We will now show that the above proposition almost characterizes the class of truthful VCG-based mechanisms for the combinatorial auction problem.

Notation: We let $\tilde{V}$ denote the space of all types $v=\left(v^{1}, \ldots, v^{n}\right)$ such that for any two different allocations $x$ and $y, g(v, x) \neq g(v, y)$. (Recall that $g($.$) denotes the total welfare.)$

It is not difficult to see that $\tilde{V}$ contains almost all the types, i.e. $V-\tilde{V}$ has a measure zero in $V$.

Theorem 3.2 If a VCG-based mechanism for the combinatorial auction problem is truthful, then its output algorithm is maximal in its range at $\tilde{V}$.

Proof: Assume by contradiction that $m=(k, p)$ is truthful but $k($.$) is not maximal in its$ range at $\tilde{V}$. Since the functions $h^{i}($.$) do not affect the truthfulness of the mechanism, we$ can assume that they are all zero, i.e., we assume that for all $i, p^{i}(w)=\sum_{j \neq i} w^{j}(k(w))$. According to Lemma 2.1, the utility of each agent $i$ equals $v^{i}(k(w))+\sum_{j \neq i} w^{j}(k(w))=$ $g\left(\left(v^{i}, w^{-i}\right), k(w)\right)$.

Let $\mathcal{O}$ denote the range of $k($.$) at \tilde{V}$ and let $v \in \tilde{V}$ be a type such that $k(v)$ is not optimal over $\mathcal{O}$. Let $y=\arg \max _{o \in \mathcal{O}} g(v, o)$ be the optimal allocation among $\mathcal{O}$. Note that 
from the definition of $\tilde{V}, y$ is unique. Finally, let $w \in \tilde{V}$ be a type such that $y=k(w)$. Such a type exists since $y$ is in the range of the algorithm.

Define a type vector $z$ by

$$
z^{i}(s)= \begin{cases}v^{i}(s) & \text { if } s \nsupseteq y^{i} \\ \alpha & \text { if } s \supseteq y^{i}\end{cases}
$$

where $\alpha$ stands for a sufficiently large number. In other words, each agent $i$ strongly desires the set $y^{i}$. Apart from this, $v^{i}$ and $z^{i}$ are identical. We assume that $z \in \tilde{V}$. Otherwise we could add sufficiently small "noise" $\epsilon^{i}(s)$ to $z$ such that all the claims below remain true.

We will show that $z$ "forces" the algorithm to output $y$. We will then show that if the algorithm outputs $y$ when the type is $z$, it must also output $y$ when the type is $v-\mathrm{a}$ contradiction.

Lemma $3.3 y=k(z)$.

Proof: Define a sequence of type vectors by:

$$
\begin{aligned}
& w_{0}=\left(w^{1}, \ldots, w^{n}\right) \\
& w_{1}=\left(z^{1}, w^{2}, \ldots, w^{n}\right) \\
& w_{2}=\left(z^{1}, z^{2}, w^{3}, \ldots, w^{n}\right) \\
& \vdots \\
& w_{n}=\left(z^{1}, \ldots, z^{n}\right) .
\end{aligned}
$$

In other words, every agent in turn moves from $w^{i}$ to $z^{i}$. We assume that $w_{j} \in \tilde{V}$ for all $j$. It is not difficult to see that $z$ can be modified by adding small noise to it, in a way that guarantees the above.

Claim $3.4 k\left(w_{1}\right)=y$.

Proof: Assume by contradiction that this is false. From the definition of $\tilde{V}$ we obtain that $g\left(w_{1}, k\left(w_{1}\right)\right) \neq g\left(w_{1}, y\right)$.

Consider the case where agent 1's type is $z^{1}$ and the types of the others are $w^{2}, \ldots, w^{n}$. By declaring $w^{1}$, agent 1 can force the algorithm to decide on $y$. Since the mechanism is truthful, it must be that $g\left(w_{1}, k\left(w_{1}\right)\right)>g\left(w_{1}, y\right)$.

Since $\alpha$ is large, it must be that $k^{1}\left(w_{1}\right) \supseteq y^{1}$ (i.e., agent 1 gets all the items it gets when its type is $\left.w^{1}\right)$. Thus, from the definition of $z^{1}$, we obtain $\alpha+\sum_{j=2}^{n} w^{j}\left(k\left(w_{1}\right)\right)>$ $\alpha+\sum_{j=2}^{n} w^{j}(y)$. Because, due to the free disposal assumption, $w^{1}\left(k\left(w_{1}\right)\right) \geq w^{1}(y)$, we obtain that $w^{1}\left(k\left(w_{1}\right)\right)+\sum_{j=2}^{n} w^{j}\left(k\left(w_{1}\right)\right)>w^{1}(y)+\sum_{j=2}^{n} w^{j}(y)$ (even when $z$ is perturbed). Thus, $g\left(w_{0}, k\left(w_{1}\right)\right)>g\left(w_{0}, y\right)$.

Therefore, when the type of agent 1 is $w^{1}$, it is better off declaring $z^{1}$, forcing the mechanism to output $k\left(w_{1}\right)$. This contradicts the truthfulness of the mechanism.

Similarly, by induction on $j$, we obtain that $k\left(w_{j}\right)=y$ for all $j$, and in particular for $w_{n}=z$. This completes the proof of Lemma 3.3.

We will now show that $k(z)=y$ implies that $k(v)=y-$ a contradiction. Consider the following sequence of type vectors: 


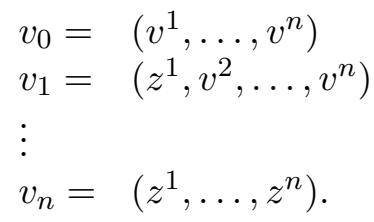

In other words, every agent in turn, moves from $v^{i}$ to $z^{i}$. Again we can choose $z$ such that all $v_{j}$ s are in $\tilde{V}$.

Claim 3.5 For all $v_{j}$, y maximizes $g$ on $\mathcal{O}$.

Proof: We will show this for $v_{1}$. The proof for $j>1$ follows from a similar argument. Assume by contradiction that $x \neq y$ maximizes the welfare for $v_{1}$. Since $\alpha$ is arbitrarily large it must be that $x^{1} \supseteq y^{i}$. Consequently, in both cases agent 1's valuation equals $\alpha$.

Recall that $y$ uniquely maximizes $g$ on $\mathcal{O}$ for $v_{0}$. Thus, for every allocation $x \neq y$, we have $v^{1}(y)+\sum_{j=2}^{n} v^{j}(y)>v^{1}(x)+\sum_{j=2}^{n} v^{j}(x)$. Therefore, $\alpha+\sum_{j=2}^{n} v^{j}(y)>\alpha+\sum_{j=2}^{n} v^{j}(x)$. But the left hand side equals $g\left(v_{1}, y\right)$ and the right hand side equals $g\left(v_{1}, x\right)$. Thus, $g\left(v_{1}, y\right)>$ $g\left(v_{1}, x\right)$ - contradiction.

Claim $3.6 k\left(v_{n-1}\right)=y$.

Proof: We showed that $k\left(v_{n}\right)=y$. (Recall that $v_{n}=z$.) We also showed that $y$ uniquely maximizes $g\left(v_{n-1},.\right)$. Let $x_{n-1}=k\left(v_{n-1}\right)$. Assume by contradiction that $x_{n-1} \neq y$. According to Lemma 2.1, the utility of agent $n$ when it is truthful is $g\left(v_{n-1}, x_{n-1}\right)$. Thus, when agent $n$ 's type is $v^{n}$, it is better off declaring $z^{n}$ obtaining a utility of $g\left(v_{n-1}, y\right)$. This contradicts the truthfulness of the mechanism.

Similarly, by downward induction on $j$, we obtain that $k\left(v_{0}\right)=y$. But $v_{0}=v$ and we assumed that $k(v) \neq y-$ a contradiction. This completes the proof of Theorem 3.2.

Remarks The above theorem characterizes the output algorithms that could be incorporated into truthful VCG-based mechanisms on all but a zero-measured subset of the types. This characterization holds even when the set of possible types is discrete (under the mild condition that the type vector $z$ can be defined such that the agents are not indifferent between allocations). The theorem gives rise to several interesting algorithmic and combinatorial questions. For example, given an approximation factor $c \leq 1$, what is the minimal size of a sub-family $\mathcal{O} \subseteq O$ such that for every $v, \max _{y \in \mathcal{O}} g(v, y) \geq c \cdot g_{\text {opt }}(v)$ ? A limited version of this question was analyzed by Holzman et al., 2004 and Holzman \& Monderer, 2004 .

Corollary 3.7 Consider a VCG-based mechanism for a combinatorial auction with an output algorithm $k$. If the mechanism is truthful, there exists an output algorithm $\tilde{k}$, maximal in its range, such that for every $v, g(v, k(v))=g(v, \tilde{k}(v))$.

Proof: Let $\mathcal{O}$ denote the range of $k$ on $\tilde{V}$, and define another algorithm that is optimal in its range by $\forall v, \tilde{k}(v) \in \arg \max _{o \in \mathcal{O}}$. According to Proposition 3.1, a VCG mechanism 
based on $\tilde{k}$ is truthful. Consider the case where the agents are truthful. Recall that the utility of all agents is determined by the resulting total welfare. Thus, it is not difficult to see that the welfares $g(v, k(v))$ and $g(v, \tilde{k}(v))$ must be continuous in $v$. Two continuous real functions, which are identical on a dense subspace, are identical on the whole space and thus the corollary follows.

We now show that non-optimal truthful VCG-based mechanisms suffer from the following disturbing abnormal behavior:

Definition 10 (reasonable mechanism) A mechanism for combinatorial auctions is called reasonable if whenever there exists an item $j$ and an agent $i$ such that:

1. For all $S$, if $j \notin S$, then $v^{i}(S \cup\{j\})>v^{i}(S)$, and,

2. For every agent $l \neq i, \forall S, v^{i}(S \cup\{j\})=v^{i}(S)$,

then $j$ is allocated to agent $i$.

Simply put, in situations where only one agent desires an item, that agent gets it.

Theorem 3.8 Any non-optimal truthful VCG-based mechanism for combinatorial auctions is not reasonable.

Proof: Consider such a mechanism $m$. According to Corollary 3.7 there exists an equivalent mechanism $\tilde{m}=(\tilde{k}, p)$, which is optimal in its range. Since $\tilde{m}$ must also be sub-optimal, there exists at least one partition $s=\left(s^{1}, \ldots, s^{n}\right)$ that is not in the range of the mechanism. Define a vector of types by:

$$
v^{i}(x)= \begin{cases}1 & \text { if } x \supseteq s^{i} \\ 0 & \text { otherwise. }\end{cases}
$$

In other words, each agent $i$ wants a single set $s^{i}$, and no two agents want the same item (as the sets are disjoined). Since $s$ is not in the range of $\tilde{m}$ it must be that $\tilde{k}(v) \neq s$. Since $s$ is strictly optimal, $k(v)$ must also be suboptimal. Hence, there exists at least one agent $i$ that does not get $s^{i}$. In particular, there exists at least one item $j \in s^{i}$ that agent $i$ does not get. Since $i$ is the only agent that desires $j$, the theorem follows.

Corollary 3.9 Unless $P=N P$, any polynomial time truthful VCG-based mechanism for combinatorial auctions is not reasonable.

We believe that most of the "natural" allocation algorithms (e.g., linear programming relaxations, algorithms that greedily allocate items to agents, local search algorithms) will not yield the above anomaly. In particular, we presume that when each agent wants a single subset of items and these subsets are disjoined, any such algorithm will find the optimal allocation. Thus, the above corollary suggests that it might be difficult to develop allocation algorithms that yield truthful VCG-based mechanisms.

We now show how to generalize our results to any affine-based mechanism. Given a tuple $a=\left(a_{0}, \ldots, a_{n}\right)$, we define $\tilde{V}$ to be the space of all types $v$ such that for any two different allocations $x$ and $y, g_{a}(v, x) \neq g_{a}(v, y)$. Similarly to the unweighted case, we say that an algorithm is optimal in its range with respect to $g_{a}($.$) if it always produces allocations that$ maximize $g_{a}($.$) .$ 
Theorem 3.10 Consider an affine-based mechanism for the combinatorial auction problem defined by an allocation algorithm $k($.$) , and a tuple a=\left(a_{0}, \ldots, a_{n}\right)$. If the mechanism is truthful, then $k($.$) is maximal in its range with respect to g_{a}($.$) at \tilde{V}$.

Proof: (sketch) The proof is similar to the proof of Theorem 3.2 and we thus only sketch it. Define $\tilde{V}$ and $\mathcal{O}$ similarly but w.r.t. the affine transformation $g_{a}($.$) . Assume by contradiction$ that there exists a type vector $v$ such that $k(v)$ is not optimal in $\mathcal{O}$. Let $y$ be the optimal allocation in the range $\mathcal{O}$, and $w \in \tilde{V}$ such that $k(w)=y$. According to Lemma 2.2, the utility of each agent is maximized with the weighted welfare $g_{a}\left(\left(v^{i}, w^{-i}\right),.\right)$. Thus, it is possible to proceed along the lines of the proof of Theorem 3.2: Define a type vector $z$ similarly; then, start from $w$ and gradually transform all agents to $z$ and conclude that $k(z)=y$; then gradually transform all agents from $z$ to $v$ and show that $k(v)=y$, i.e., $-\mathrm{a}$ contradiction.

Open Questions We currently do not know whether theorems similar to Theorem 3.2 hold when the valuations are bounded. Moreover, we do not know how to get rid of the usage of $\tilde{V}$. Thus, we do not preclude the possibility that Corollary 3.7 will not hold when the space of possible types is discrete. We also do not know whether they hold when the allocation algorithm is randomized or whether Bayesian versions of our theorems apply to the expected externality mechanism (d'Aspremont \& Gerard-Varet, 1979) (an analog of VCG in the Bayesian model). We leave this to future research. We conjecture that similar theorems apply to many other mechanism design problems.

\subsection{Truthful VCG-based Mechanisms for Cost Minimization Problems}

We now show that for many natural cost minimization problems, any truthful VCG-based mechanism is either optimal or produces results that are arbitrarily far from the optimal. We start with a sample problem.

Multicast transmissions: A communication network is modeled by a directed graph $G=(V, E)$. Each edge $e$ is a privately owned link. The cost $t_{e}$ of sending a message along that edge is privately known to its owner. Given a source $s \in V$ and a set $T \subseteq V$ of terminals, the mechanism must select a subtree rooted in $s$ that covers all the terminals. The message is then broadcasted along this tree. We assume that no agent owns a cut in the network.

Naturally, the goal of the mechanism is to select, among all possible trees, a tree $R$ that minimizes the total cost: $\sum_{e \in R} t_{e}$. The goal of each agent is to maximize its own profit:

$p^{i}-\sum_{(e \in R}$ owned by $\left.i\right) t_{e}$. It is not difficult to see that this is a utilitarian mechanism design problem.

This example was introduced by Feigenbaum et al., 2000 (using a different model). It is motivated by the need to broadcast long messages (e.g., movies) over the Internet. We now generalize this example.

\section{Definition 11 (cost minimization allocation problem)}

A cost minimization allocation problem (CMAP) is a mechanism design problem described by: 
Type space The type of each agent $i$ is described by a vector $\left(v_{1}^{i}, \ldots, v_{m_{i}}^{i}\right)$. We let $m=$ $\sum_{i} m_{i}$. (In our multicast example $v_{e}^{i}$ corresponds to the negation of the cost $t_{e}$.)

Allowable outputs Each output is denoted by a bit vector $x=\left(x_{1}^{1}, \ldots, x_{m_{1}}^{1}, \ldots, x_{1}^{n}, \ldots, x_{m_{n}}^{n}\right) \in$ $\{0,1\}^{m}$. We denote $\left(x_{1}^{i}, \ldots, x_{m_{i}}^{i}\right)$ by $x^{i}$. There may be additional constraints on the set $O$ of allowable outputs. (In our example $x$ corresponds to a tree in the network's graph where $x_{j}^{i}$ equals 1 iff the corresponding edge is in the chosen tree.)

such that the following conditions are satisfied:

Unbounded costs If $v^{i}=\left(v_{1}^{i}, \ldots, v_{m^{i}}^{i}\right)$ describes a type for agent $i$ and $w^{i} \leq v^{i}$ (as vectors), then $w^{i}$ also describes a type.

Independence and monotonicity Each valuation $v^{i}$ depends only on $i$ 's bits $x^{i}$. (In our example, the agent valuation of a given tree depends only on its own edges in it.) If for all $j, w_{j}^{i} \leq v_{j}^{i}$, then for every output $x, w^{i}\left(x^{i}\right) \leq v^{i}\left(x^{i}\right)$.

Forcing condition For every type $v$, an allowable output $x$ and a real number $\alpha$, define a type $v[\alpha]$ by

$$
v[\alpha]_{j}^{i}= \begin{cases}v_{j}^{i} & \text { if } x_{j}^{i}=1 \\ \alpha & \text { otherwise }\end{cases}
$$

The forcing condition is satisfied if for every allowable output $y \neq x, \lim _{\alpha \rightarrow-\infty} g(t(\alpha), y)=$ $-\infty$.

Many natural decentralized task allocation problems in which the goal is to minimize the total cost under given constraints belong to this class. In particular the reader may verify that our multicast example falls into this category. Another example is the shortest path problem studied extensively in recent years (e.g., Rosenschein \& Zlotkin, 1994; Archer \& Tardos, 2002; Elkind et al., 2004).

Notation: For a type $v$ we let $g_{\text {opt }}(v)$ denote the optimal value of $g$. We denote $g(v, k(v))$ by $g_{k}(v)$.

Definition 12 (degenerate algorithm) An output algorithm $k$ is called degenerate if the ratio $r_{k}(v)=\frac{g_{k}(v)-g_{\text {opt }}(v)}{\left|g_{\text {opt }}(v)\right|+1}$ is unbounded, i.e., there exist $v$ 's such that $r_{k}(v)$ is arbitrarily large.

A degenerate algorithm is arbitrarily far from optimal, both additively and multiplicatively. Note that this should not be confused with the standard notion of an approximation ratio, as our definition corresponds to a single problem. In particular, the number of agents is fixed. We note that we do not rule out the possibility that such an algorithm will be good by some non worst case metric.

Theorem 3.11 If a VCG-based mechanism for a CMAP is truthful, then its output algorithm is either optimal or degenerate. 
Before stating the proof let us illustrate it using the multicast transmission example. Suppose that we start with a type vector that leads to a sub-optimal solution. If we raise the cost of an edge, the utility of the owner cannot increase (due to the truthfulness and Lemma 2.1). We then gradually raise the cost of all edges except the ones in the optimal tree. Still, the algorithm will have to choose a sub-optimal tree. However, the cost of any suboptimal tree is now arbitrarily high while the optimal cost remains the same.

Proof: Let $m=(k, p)$ be a non-optimal truthful VCG-based mechanism for a CMAP. As in Theorem 3.2, assume that $p^{i}(w)=\sum_{j \neq i} w^{j}(k(w))$. Let $v$ be a type vector such that $k(v)$ is not optimal and let $y=o p t(v)$ be an optimal output.

We define a type $z$ by:

$$
z_{j}^{i}= \begin{cases}v_{j}^{i} & \text { if } y_{j}^{i}=1 \\ -\alpha & \text { otherwise }\end{cases}
$$

where $\alpha$ is arbitrarily large.

Consider the type sequence:

$$
\begin{aligned}
& v_{0}=\left(v^{1}, \ldots, v^{n}\right) \\
& v_{1}=\left(z^{1}, v^{2}, \ldots, v^{n}\right) \\
& \vdots \\
& v_{n}=\left(z^{1}, \ldots, z^{n}\right) .
\end{aligned}
$$

Claim 3.12 For all $j, y=\operatorname{opt}\left(v_{j}\right)$.

Proof: By definition $y$ is optimal for $v_{0}$. Let $x \neq y$ be an allocation. From the independence condition, for all $j, g\left(v_{j}, y\right)=g\left(v_{0}, y\right)$. From the monotonicity, $g\left(v_{j}, x\right) \leq g\left(v_{0}, x\right)$. Together, $g\left(v_{j}, x\right) \leq g\left(v_{0}, x\right) \leq g\left(v_{0}, y\right)=g\left(v_{j}, y\right)$.

Claim $3.13 g\left(v_{1}, k\left(v_{1}\right)\right)<g\left(v_{1}, y\right)$

Proof: Assume by contradiction that the claim is false. Since $y$ is optimal for $v_{1}$, this means that $g\left(v_{1}, k\left(v_{1}\right)\right)=g\left(v_{1}, y\right)$. From independence, $g\left(v_{1}, y\right)=g\left(v_{0}, y\right)$. Recall that $k\left(v_{0}\right)$ is suboptimal so $g\left(v_{0}, y\right)>g\left(v_{0}, k\left(v_{0}\right)\right)$. From monotonicity (we only worsen the type of agent 1), $g\left(v_{0}, k\left(v_{1}\right)\right) \geq g\left(v_{1}, k\left(v_{1}\right)\right)$. Thus, together $g\left(v_{0}, k\left(v_{1}\right)\right) \geq g\left(v_{1}, k\left(v_{1}\right)\right)=$ $g\left(v_{1}, y\right)=g\left(v_{0}, y\right)>g\left(v_{0}, k\left(v_{0}\right)\right)$. In particular, $g\left(v_{0}, k\left(v_{1}\right)\right)>g\left(v_{0}, k\left(v_{0}\right)\right)$.

Consider the case where agent 1 's type is $v^{1}$ and the declarations of the other agents are $\left(v^{2}, \ldots, v^{n}\right)$. According to Lemma 2.1, its utility when it is truthful, equals $g\left(v_{0}, k\left(v_{0}\right)\right)$. On the other hand, when it falsely declares $z^{1}$, its utility equals $g\left(v_{0}, k\left(v_{1}\right)\right)$. Since we showed that $g\left(v_{1}, k\left(v_{1}\right)\right)>g\left(v_{0}, k\left(v_{0}\right)\right)$, this contradicts the truthfulness of the mechanism.

Similarly, we obtain that $g\left(v_{n}, k\left(v_{n}\right)\right)<g\left(v_{n}, y\right)=g\left(v_{0}, y\right)$. By the forcing condition, $g\left(v_{n}, k\left(v_{n}\right)\right) \rightarrow-\infty$ when $\alpha \rightarrow \infty$. Thus, the algorithm is degenerate.

Corollary 3.14 Unless $P=N P$, any polynomial time truthful $V C G$-based mechanism for an NP-hard CAMP is degenerate. 
Note that due to the revelation principle, the theorems in this section hold for any mechanism where the agents have dominant strategies. Similarly to Theorem 3.11, any mechanism that uses VCG payments and has a non-optimal ex-post Nash equilibrium also has equilibria which are arbitrarily far from optimal.

We now show how to generalize the theorems in this section to affine-based mechanisms.

Theorem 3.15 If an affine-based mechanism $(k, p)$ for a CMAP is truthful, then its output algorithm is either optimal or degenerate.

Proof:(sketch) The proof is almost identical to the proof of Theorem 3.11. Let $v$ be a type such that $k(w)$ is not optimal w.r.t. to the corresponding affine transformation $g_{a}$. We define a type vector $z$ similarly to Theorem 3.11 and consider a sequence of type vectors where each agent in turn changes its type from $w^{i}$ to $z^{i}$. Due to the incentive compatibility and Lemma 2.2, the utility of each agent cannot increase, meaning that the weighted welfare $g_{a}$ remains sub-optimal. Due to the forcing condition, all outputs except the optimal, have arbitrarily high cost. This means that the algorithm is degenerate.

The compensation and bonus mechanism (Nisan \& Ronen, 2001) identifies the utility of agents with the total welfare similarly to VCG, i.e., the utility of an agent can be described similarly to Lemma 2.1. Thus, all the theorems in this section can be applied to compensation and bonus mechanisms as well.

\section{Second Chance Mechanisms}

To date, affine maximization is the only known general method for the development of truthful mechanisms. Therefore, the results in the previous section do not leave much hope for the development of truthful mechanisms for many complex problems.

This section proposes a method for circumventing this problem. Consider a VCG-based mechanism. An immediate consequence of Lemma 2.1 is that the only reason for an agent to misreport its type is to help the algorithm to improve the overall result. This leads to the intuition that if the agents cannot improve upon the underlying algorithm, they can do no better than be truthful. We would like to exploit this special property of VCG-based mechanisms and construct mechanisms that are "almost" truthful.

Given any algorithm for the corresponding optimization problem we define the second chance mechanism based on it. This mechanism is a modification of the VCG-based mechanism where in addition to their valuations, the agents are allowed to submit appeal functions. An appeal function allows the agent to give the algorithm an input (vector of declared valuations) that is different from the original input but without misreporting its type. When the agents behave truthfully, the welfare obtained by the mechanism is at least as good as the one obtained by the algorithm's output.

We then formulate the rationale for truthfulness in second chance mechanisms. Informally, our argument is as follows: Under reasonable assumptions, in any situation in which the agent believes it is beneficial for it to lie to the mechanism, it is better for it to report its actual type to the mechanism and ask its appeal to check whether this lie is indeed helpful. Thus, the agent can construct a truthful strategy premised on the fact that it is not aware 
of any situation in which another strategy is better for it. We believe that this is a strong argument for truth-telling.

A generalization of our results to affine maximization and to compensation and bonus mechanisms is straightforward.

\subsection{The Mechanism}

In this section we formulate the second chance mechanism and its basic properties.

Definition 13 (appeal function) Let $V=\prod_{i} V_{i}$ denote the type space of the agents. An appeal is a partial function ${ }^{5} l: V \rightarrow V$.

The semantics of an appeal $l($.$) is: "when the agents' type vector is v=\left(v_{1}, \ldots, v_{n}\right)$, I believe that the output algorithm $k($.$) produces a better result (w.r.t. v$ ) if it is given the input $l(v)$ instead of the actual input $v$ ". An appeal function gives the agent an opportunity to improve the algorithm's output. If $v$ is not in the domain of $l($.$) , the semantics is that$ the agent does not know how to cause the algorithm to compute a better result than $k(v)$.

The second chance mechanism is defined in Figure 4.1. It is a modification of VCG that allows the agents to submit appeal functions as well.

Before the execution The manager of the mechanism publishes the outcome determination algorithm and a time limit $T$ on the computation time of each appeal.

Declaration Each agent submits a type declaration $w^{i}$ and an appeal function $l^{i}($.$) to the$ mechanism. The appeals must adhere to the specified time limit.

Allocation Let $w=\left(w^{1}, \ldots, w^{n}\right)$. The mechanism computes $k(w), k\left(l^{1}(w)\right), \ldots, k\left(l^{n}(w)\right)$ and chooses among these outputs the one that maximizes the total welfare (according to $w)$.

Payment Let $\hat{o}$ denote the chosen output. The mechanism calculates the payments according to the VCG formula: $p^{i}=\sum_{j \neq i} w^{j}(\hat{o})+h^{i}\left(w^{-i}, l^{-i}\right)$ (where $h^{i}($.$) is any real$ function).

Figure 1: The Second Chance Mechanism

Remarks The agents send programs that represent their appeal functions to the mechanism. These programs are then executed by the mechanism. The mechanism can terminate the computation of each appeal after $T$ units of computation time (and refer to the vector of declarations $w$ as if it is not in the appeal's domain). Thus, we can assume w.l.o.g. that all appeals adhere to the given time limit. A discussion on the choice of the time limit and alternative representations of the appeal functions appears in Section 4.3. We believe that it is possible to construct software tools and APIs that will make the formulation of the appeals an easy task.

5. A function $f: D \rightarrow R$ is called partial if its domain is a subset of $D$, i.e. if $\operatorname{Dom}(f) \subseteq D$. 
The functions $h^{i}($.$) do not play any role in the agents' considerations as every h^{i}($.$) is$ independent of $i$ 's actions. Until Section 4.4 it is possible to simply assume that $h^{i}(.) \equiv 0$ for all $i$. In Section 4.4 we will use these functions in order to satisfy individual rationality.

Definition 14 (truthful action) An action in the second chance mechanism is a pair $\left(w^{i}, l^{i}\right)$ where $w^{i}$ is a type declaration and $l^{i}($.$) is an appeal function. An action is called$ truthful if $w^{i}=v^{i}$.

The following observation is a key property of the mechanism.

Proposition 4.1 Consider a second chance mechanism with an output algorithm $k$. For every type vector $v=\left(v^{1}, \ldots, v^{n}\right)$, if all agents are truth-telling, $g(v, \hat{o}) \geq g(w, k(v))$.

In other words, when the agents are truth-telling, the result of the mechanism is at least as good as $k(v)$. The proof is immediate from the definition of the mechanism. We now formulate an analog of Lemma 2.1. The proof is similar to the lemma's proof and is henceforth omitted.

Lemma 4.2 (second chance utility) Consider a second chance mechanism. Let $\hat{o}$ be the chosen output. The utility of agent $i$ equals $g\left(\left(v^{i}, w^{-i}\right), \hat{o}\right)+h^{i}\left(w^{-i}, l^{-i}\right)$.

Therefore, informally, it is beneficial for an agent to declare $w^{i} \neq v^{i}$ only if it either helps the output algorithm $k$ (.) to compute a better result (w.r.t. $\left.\left(v^{i}, w^{-i}\right)\right)$ or it helps one of the appeals of the other agents.

Note that lying to a second chance mechanism may harm an agent in two ways. First, it can damage the output algorithm $k($.$) . Second, it can cause the mechanism to measure$ the welfare according to a wrong type vector and thus cause it to choose an inferior output.

Notation: We say that a second chance mechanism is T-limited if the time limit it specifies is $T$. Similarly, an algorithm is called T-limited if its computational time never exceeds $T$ units of computation.

The following proposition is obvious.

Proposition 4.3 Consider a T-limited second chance mechanism. If the output algorithm of the mechanism is also T-limited, the overall computational time of the mechanism is $O(n T)$.

\subsubsection{A TOY EXAMPLE}

Consider a combinatorial auction of two items. A type of an agent is a 3-tuple representing its value for every non empty subset of items. Suppose that agent $i$ values the pair of items at $\$ 3$ million but values every single item at $\$ 1$ million. Its type is, therefore, $v^{i}=$ 
$\{3,1,1\}$. Suppose that the agent notices that the allocation algorithm often produces better allocations if it declares $w^{i}=\{3,0,0\}$ (i.e., it hides its willingness to accept only one item). In a VCG-based mechanism the agent may prefer to declare $w^{i}$ instead of its actual type. This might cause two problems:

1. Even when the others are truthful, there may be many type vectors $v^{-i}$ belonging to the other agents, for which declaring $w^{i}$ damages the chosen allocation, i.e., $g\left(\left(v^{i}, w^{-i}\right), k\left(\left(w^{i}, w^{-i}\right)\right)\right)<g\left(\left(v^{i}, w^{-i}\right), k\left(\left(v^{i}, w^{-i}\right)\right)\right)$.

2. Even when this is not the case and every agent $i$ chooses a declaration $w^{i}$ such that $g\left(\left(v^{i}, w^{-i}\right), k\left(\left(w^{i}, v^{-i}\right)\right)\right) \geq g\left(\left(v^{i}, w^{-i}\right), k(w)\right)$, it may be that according to the actual type vector $v$ the output $k(w)$ may be inferior to $k(v)$ (i.e., $g(v, k(w))<g(v, k(v)))$.

The second chance mechanism enables the agent to check whether declaring a falsified type would yield a better result. Instead of declaring $w^{i}=\{3,0,0\}$, the agent can declare its actual type and define its appeal as $l^{i}\left(w^{\prime}\right)=\left(w^{i}, w^{\prime-i}\right)$. In this way the agent enjoys both worlds. In cases where the falsified type is better, the mechanism will prefer $k\left(\left(w^{i}, w^{-i}\right)\right)$ over $k\left(\left(v^{i}, w^{-i}\right)\right)$. In cases where the truthful declaration is better, the mechanism will prefer $k\left(\left(v^{i}, w^{-i}\right)\right)$. Note that the mechanism allows an appeal to modify not only the declaration of the agent that submitted it but also the whole vector of declarations. This will allow us to provide a strong argument for truth-telling.

Possible Variants of the Second Chance Mechanism One alternative definition of the mechanism is to let the agents submit outcome determination algorithms instead of appeals. It is possible to apply reasoning similar to ours to this variant. However, formulating such output algorithms might be a demanding task for many applications. There are also other more delicate differences.

Another possibility is to define a multi-round variant of the mechanism. In the first round the agents submit type declarations $w$. Then, at each round, each agent gets the chance to improve the allocation found by the algorithm $k(w)$. The mechanism terminates when no agent improves the current allocation. The strategy space of multi-round mechanisms is very complex. Yet, under myopic behavior (Parkes, 1999), arguments similar to ours can be used to justify truthful behavior. Such arguments may explain the relative success of ad hoc mechanisms such as iterative VCG (IVG) and AUSM ${ }^{6}$ reported by Banks et al., 1989 .

Standard Equilibria in Second Chance Mechanisms The second chance mechanism uses VCG payments and, therefore, the theorems of the first part of the paper apply to it. From Lemma 4.2, a vector of truthful actions is an ex post equilibrium if and only if the resulting allocation $\hat{o}$ is optimal in the range of the algorithm. Moreover, consider agent $i$ and let $\left(w^{-i}, l^{-i}\right)$ be a set of actions of the other agents. $\left(w^{i}, l^{i}\right)$ is a best response for the agent if and only if the resulting allocation $\hat{o}$ is optimal in the range of the underlying algorithm with respect to $\left(v^{i}, w^{-i}\right)$. At least intuitively, finding such a response is at least as

6. Both mechanisms are in the spirit of our second chance mechanism, as they let the agents improve the allocation. The actual rules of these mechanisms are complicated and are described by Banks et al., 1989 . 
hard as finding an allocation that is optimal in the range of the algorithm. Thus, one should not expect the agents to follow equilibrium strategies in the traditional sense. We argue that similar arguments can be made for every game in which computing the best response is computationally difficult. Hence, an argument that takes into account the agents' own limitations is required. We note that we did not succeed in finding natural complexity limitations under which truth-telling is an equilibria for the agents. We leave this as an intriguing open problem.

\subsection{The Rationale for Truth-telling}

As we noted, standard equilibria should not be expected in second chance mechanisms. This section formulates the rationale for truth-telling under these mechanisms. We first introduce the notion of feasibly dominant actions ${ }^{7}$ which takes into account the fact that the agents' capabilities are limited. We then demonstrate that under reasonable assumptions about the agents, truthful, polynomial time, feasibly dominant actions exist.

\subsubsection{Feasible Truthfulness}

The basic models of equilibria in game theory are justified by the implicit assumption that the agents are capable of computing their best response functions. In many games, however, the action space is huge and this function is too complex to be computed, even approximately within a reasonable amount of time. In such situations the above assumption seems no longer valid.

In this section we re-formulate the concept of dominant actions under the assumption that agents have a limited capability of computing their best response. Our concept is meant to be used in the context of one stage games, i.e. games in which the agents choose their actions without knowing anything about the others' choices. The second chance mechanism is a one stage-game. In a nutshell, an action is feasibly dominant if the agent is not aware of any situation (a vector of the other agents' actions) where another action is better for it.

Notation: We denote the action space of each agent $i$ by $A^{i}$. Given a tuple $a=\left(a^{1}, \ldots, a^{n}\right)$ of actions chosen by the agents, we denote the utility of agent $i$ by $u^{i}(a)$.

Definition 15 (revision function) $A$ revision function of agent $i$ is any partial function of the form $b^{i}: A^{-i} \rightarrow A^{i}$.

The semantics of $b^{i}\left(a^{-i}\right)$ is "If I knew that the actions of the others are $a^{-i}$, I would choose $b^{i}\left(a^{-i}\right)$ (instead of $\left.a^{i}\right)$ ". A revision function captures all the cases where the agent knows how it would like to act if it knew the others' actions. Note that optimal revision functions are standard best-response functions. When a vector of actions $a^{-i}$ does not belong to the domain of $b^{i}($.$) , the semantics is that the agent prefers to stick to its action.$

Definition 16 (feasible non-regret) Let $i$ be an agent, $b^{i}($.$) its revision function, and$ $a^{-i}$ a vector of actions for the other agents. An action $a^{i}$ satisfies the feasible non-regret

7. We make a standard distinction between an action and a strategy - a mapping from the agent's type to its action. 
condition (w.r.t. $a^{-i}$ and $\left.b^{i}\right)$, if either $a^{-i}$ is not in the domain of $b^{i}$ or $u^{i}\left(\left(b^{i}\left(a^{-i}\right), a^{-i}\right)\right) \leq$ $u^{i}(a)$.

In other words, other actions may be better against $a^{-i}$, but the agent is unaware of them or cannot compute them when choosing its action.

When the revision function of the agent is optimal, a feasible non-regret is equivalent to the standard non-regret (best response) condition.

Definition 17 (feasibly dominant action) Let $i$ be an agent, $b^{i}($.$) its revision function.$ An action $a^{i}$ is called feasibly dominant (w.r.t. $\left.b^{i}().\right)$ if for every vector $a^{-i}$ of the actions of the other agents, $a^{i}$ satisfies the feasible non-regret condition (w.r.t. $a^{-i}$ and $b^{i}$ ).

Put differently, an action $a^{i}$ is feasibly dominant if (when choosing its action) the agent is not aware of any action $a^{i}$ and any vector $a^{-i}$ of the actions of the other agents, such that it is better off choosing $a^{i}$ when the others choose $a^{-i}$. A dominant action is always feasibly dominant. When the revision function is optimal, a feasibly dominant action is dominant.

Example In order to demonstrate the concept of feasibly dominant actions consider a chess match in which Alice and Bob submit computer programs that play on their behalf. Currently, of course it is not known how to compute an equilibrium in chess and therefore standard equilibria are not relevant for the analysis of such a game. A program $a_{A}$ is feasibly dominant for Alice if she is not aware of any possible program of Bob against which she is better off submitting another program.

Definition 18 (feasibly truthful action) An action $a^{i}$ in the second chance mechanism is called feasibly truthful if it is both, truthful and feasibly dominant.

\subsubsection{NATURAL REVISION FUNCTIONS THAT GIVE RISE TO FEASIBLY TRUTHFUl ACTIONS}

Beforehand we showed that when the agents are truthful, the total welfare is at least $g(v, k(v))$. We also argued that if a feasibly truthful action is available, the agent has a strong incentive to choose it. This subsection demonstrates that under reasonable assumptions about the agents, polynomial time feasibly truthful actions do exist.

Notation: We let $\perp$ denote the empty appeal. By $(w, \perp)$ we denote an action vector where the declaration of each agent $i$ is $w^{i}$ and all the appeals are empty.

Definition 19 (appeal-independent revision function) A revision function $b^{i}($.$) is$ called appeal independent if every vector in its domain includes only empty appeals, i.e. for all $a^{-i} \in \operatorname{dom}\left(b^{i}\right)$, there exists a vector $w^{-i}$ such that $a^{-i}=\left(w^{-i}, \perp\right)$.

We say that an appeal independent function is T-limited if its own computational time is bounded by $T$ and so is every appeal function in its range.

The class of appeal-independent revision functions represents agents that only explore the output algorithm (or alternatively, base their choice of action solely on the output algorithm). This approach seems reasonable as the space of appeals of the other agents is 
huge, with no apparent structure. At least intuitively, it seems unreasonable that an agent will be able to lie in a way that will improve the result of the appeals of the other agents with significant probability. Moreover, as we commented, an agent has an obvious potential loss from misreporting its type.

Theorem 4.4 Consider a second chance mechanism with a T-limited output algorithm. Suppose that an agent has a T-limited appeal-independent revision function. For every $T^{\prime}=\Omega(T)$, if the mechanism is $T^{\prime}$-limited, the agent has a feasibly truthful action.

Proof: Let $b^{i}($.$) be the agent's revision function. Define an appeal l^{i}($.$) as follows. For$ every vector $w^{-i}$, let $\left(w^{i}, \tau^{i}\right)=b^{i}\left(\left(w^{-i}, \perp\right)\right)$. Let $w=\left(w^{i}, w^{-i}\right)$. Consider the outputs $o_{1}=k(w)$ and $o_{2}=k\left(\tau^{i}(w)\right)$. We define $l^{i}(w)$ to be the better of the two outputs, i.e., $l^{i}(w)=\arg \max _{j=1,2} g\left(\left(v^{i}, w^{-i}\right), o_{j}\right)$. Intuitively, $l^{i}($.$) checks whether declaring w^{i}$ is helpful for the agent.

Claim 4.5 $a^{i}=\left(v^{i}, l^{i}\right)$ is feasibly truthful.

Proof: If it is not, there exists a vector $a^{-i}=\left(w^{-i}, \perp\right)$ in the domain of $b^{i}($.$) such that$ $u\left(a^{i}, a^{-i}\right)<u\left(b^{i}\left(a^{-i}\right), a^{-i}\right)$. Let $b^{i}\left(a^{-i}\right)=\left(w^{i}, \tau^{i}\right)$. Recall that according to Lemma 4.2, the agent's utility is equivalent to the total welfare $g\left(\left(v^{i}, w^{-i}\right), o\right)$ of the chosen output $o$ (up to adding $h^{i}($.$) , which is independent of the agent's actions).$

Consider the case when the agent's action is $b^{i}\left(a^{-i}\right)$. Let $\hat{o}$ denote the chosen output in this case. According to the definition of the mechanism, $\hat{o}$ is taken from the set $\left\{o_{1}, o_{2}\right\}$ and the welfare is measured according to the declaration $w$.

When the agent chooses the truthful action $a^{i}$, the output (denoted $\tilde{o}$ ) is chosen from the outputs $o_{0}=k\left(\left(v^{i}, w^{-i}\right)\right)$ (from the definition of the mechanism), and both, $o_{1}, o_{2}$ (from the definition of $l^{i}$ ). This is a superset of the set outputs of the first case. Moreover, the output is chosen according to the "right" type vector $\left(v^{i}, w^{-i}\right)$. Thus, $g\left(\left(v^{i}, w^{-i}\right), \tilde{o}\right) \geq$ $g\left(\left(v^{i}, w^{-i}\right), \hat{o}\right)$, implying that the agent has a higher utility in the second case - a contradiction.

It remains to show that $l^{i}($.$) is \Omega(T)$-limited. This is obvious as both, $k($.$) and \tau^{i}($.$) are$ $T$-limited. This completes the proof of the theorem.

Given the agent's revision function, it is easy to construct the appeal $l^{i}($.$) defined above (i.e.,$ construct the program that computes it). Thus, if the agent has such an appeal independent function, it can guarantee itself a feasibly dominant action.

A more general class of revision functions can be found in the Appendix. Interestingly, there is a tradeoff between the generality of the class and the time limit, which suffices for feasible truthfulness.

\subsection{Remarks on the Choice of the Time Limit}

Sections 4.2.2 and A.1 demonstrate two natural classes of revision functions under which the agents have polynomial time feasibly truthful actions. We do not claim that every revision function in practice will fall into these categories. Yet, it is plausible that this will be the case in many applications. In general, there exists a tradeoff between the generality 
of the class of the revision functions and the time limit required for feasible truthfulness. In particular, without any time limit, submitting an optimal appeal is dominant. On the other hand, it is plausible that small time limits will suffice in practice. We leave a more comprehensive study of this tradeoff to future research.

An interesting future direction is to develop representations of the appeal functions that relate the time limit imposed on each agent to its actual revision function. One possibility is to represent the appeals by decision trees where the agents are required to supply for each leaf $\alpha$, a type vector $v_{\alpha}$, such that the algorithm's result is strictly improved when it is given $l\left(t_{\alpha}\right)$ instead of the actual input $v_{\alpha} . v_{\alpha}$ proves to the mechanism that the computational time required to compute the leaf $\alpha$ is indeed needed in order to represent the agent's revision function. A related possibility is to allow the agent to purchase additional computational time.

Currently, we do not know whether every polynomial class of revision functions guarantees the existence of polynomial feasibly truthful actions. If an agent has substantial knowledge of the appeal space of the other agents, it may be able to find a falsified declaration that causes "typical" appeals to produce better results. In such a case, it may be beneficial for an agent to lie. We do not know whether such knowledge will exist in practice. If yes, it may be possible to overcome this by allowing the agents to submit meta-appeals, i.e., functions that let the agents modify the input of the appeals of the other agents. We leave this to future research.

\subsection{Obtaining Individual Rationality}

A basic desirable property of mechanisms is that the utility of a truthful agent is guaranteed to be non-negative (individual rationality). In this section we construct a variant of second chance mechanisms that satisfies this property.

Let $g_{\text {opt }}(v)$ denote the optimal welfare obtained when the type vector is $v$. We shall assume that for each agent $i$, there exists a type $\underline{v}^{i}$ such that for every $v=\left(v^{1}, \ldots, v^{n}\right)$, $g_{\text {opt }}\left(\left(\underline{v}^{i}, v^{-i}\right)\right) \leq g_{\text {opt }}(v)$. We call such a type the lowest. In a combinatorial auction for example, the lowest type is defined by the zero valuation $\underline{v}^{i}(s)=0$ for every combination $s$ of items.

The Clarke mechanism (Clarke, 1971) is a VCG mechanism in which $h^{i}\left(w^{-i}\right)=-g_{\text {opt }}\left(\underline{v}^{i}, w^{-i}\right)$, i.e., $p^{i}(w)=\sum_{j \neq i} w^{j}(o p t(w))-g_{o p t}\left(\underline{v}^{i},\left(w^{-i}\right)\right)$. In other words, each agent pays the welfare loss it causes to the society. Thus, it is natural to define the payment of a VCG-based mechanism as $\sum_{j \neq i} w^{j}(\operatorname{opt}(w))-g\left(\left(\underline{v}^{i}, w^{-i}\right), k\left(\left(\underline{v}^{i}, w^{-i}\right)\right)\right)$.

Like truthfulness, individual rationality may not be preserved when the optimal algorithm in the Clarke mechanism is replaced by a sub-optimal one. In order to fix this we need to ensure that the result of the algorithm will not improve when the declaration $w^{i}$ is replaced by the lowest type $\underline{v}^{i}$.

Definition 20 (lowest type closure) Given an allocation algorithm $k(w)$ we define its lowest type closure $\tilde{k}$ as the best allocation (according to $w$ ) among the outputs $\left(k(w), k\left(\left(\underline{v}^{1}, w^{-1}\right)\right), \ldots, k\left(\left(\underline{v}^{n}, w^{-}\right.\right.\right.$

Since $\tilde{k}($.$) calls k()$.$n times, if k$ is $T$-limited, then $\tilde{k}$ is $O(n T)$-limited.

Claim 4.6 For every $w, g(w, \tilde{k}(w)) \geq g\left(\left(\underline{v}^{i}, w^{-i}\right), k\left(\left(\underline{v}^{i}, w^{-i}\right)\right)\right)$. 
Proof: Since $k\left(\left(\underline{v}^{i}, w^{-i}\right)\right)$ is a candidate output that $\tilde{k}$ tests, $g(w, \tilde{k}(w)) \geq g\left(w, k\left(\left(\underline{v}^{i}, w^{-i}\right)\right)\right)$. Given the definition of $\underline{v}^{i}, g\left(w, k\left(\left(\underline{v}^{i}, w^{-i}\right)\right)\right) \geq g\left(\left(\underline{v}^{i}, w^{-i}\right), k\left(\underline{v}^{i}, w^{-i}\right)\right)$, the claim follows.

Definition 21 (second chance-IR) Given an allocation algorithm $k(w)$ and a time limit $T$ we define the corresponding second chance-IR mechanism as the second chance mechanism with output algorithm $\tilde{k}($.$) , time limit T$, and for every agent $i, h^{i}\left(w^{-i}\right)=-g\left(\left(\underline{v}^{i}, w^{-i}\right), k\left(\left(\underline{v}^{i}, w^{-i}\right)\right)\right)$.

The utility of a truthful agent in the above mechanism equals $u^{i}=g(w, \hat{o})-g\left(\left(\underline{v}^{i}, w^{-i}\right), k\left(\left(\underline{v}^{i}, w^{-i}\right)\right)\right) \geq$ $g(w, k(w))-g\left(\left(\underline{v}^{i}, w^{-i}\right), k\left(\left(\underline{v}^{i}, w^{-i}\right)\right)\right) \geq 0$. Therefore, the mechanism satisfies individual rationality.

\section{Conclusion and Future Research}

This paper studies VCG mechanisms in which the optimal outcome determination algorithm is replaced by some sub-optimal but computationally tractable algorithm. The first part of the paper shows that for a wide range of problems, such mechanisms lose the game theoretic virtues of their optimal counterparts. Similar results hold for affine maximization. These results do not leave much hope for the development of polynomial time truthful mechanisms for many problems of high complexity.

The second part of the paper proposes a general method for overcoming the difficulty of constructing truthful mechanisms. Given any algorithm for the underlying optimization problem we define the second chance mechanism based on it. We demonstrate that under reasonable assumptions about the agents, truth-telling is still the rational strategy for the agents. When the agents are truthful, the welfare obtained by the mechanism is at least as good as the one obtained by the underlying algorithm.

Successful implementation of second chance mechanisms relies on several tools to be developed - in particular, tools for the description of valuations and appeal functions. These "engineering" issues require further exploration.

It is important to stress that the second chance method has not yet been tested. In particular, the truthfulness of the agents should be validated experimentally. On the other hand, we believe that in practice, small time limits on the agents' appeals are likely to guarantee the truthfulness of the agents. Several questions regarding the payment properties of second chance mechanisms are open. We leave them for future research.

Several open questions, which directly stem from this work, are raised within the body of the paper.

\section{Acknowledgments}

We thank Abraham Newman and Motty Perry for helpful discussions at various stages of this work. We thank Ron Lavi, Ahuva Mu'alem, Elan Pavlov, Inbal Ronen, and the anonymous reviewers for comments on earlier drafts of this paper. Noam Nisan was supported by grants from the Israel Science Foundation and from the USA-Israel Binational Science Foundation. Amir Ronen was supported in part by grant number 969/06 from the Israel Science Foundation. A preliminary version of this paper appeared in the proceedings of the 3rd ACM Conference on Electronic Commerce (EC' 01). 


\section{Appendix A. $d$-bounded Revision Functions}

The class of $d$-bounded revision functions represents agents that, in addition to the output algorithm, explore a polynomial family of potential appeals of the other agents. This class is a generalization of $d$-limited appeal-independent functions.

Definition 22 ( $d$-bounded revision function) We say that a revision function $b^{i}($.$) is$ $d$-bounded if the following hold:

1. The revision function $b^{i}($.$) is O\left(n^{d}\right)$-limited.

2. Let

$L=\left\{l_{j} \mid \exists l^{-i,-j}, w^{-i}\right.$ s.t. $\left.\left(w^{-i},\left(l^{i}, l^{-i,-j}\right)\right) \in \operatorname{Dom}\left(b^{i}\right)\right\} \bigcup\left\{l_{i} \mid \exists\left(w^{-i}, l^{-i}\right), w^{i}\right.$ s.t. $\left.\left(w^{i}, l^{i}\right)=b^{i}\left(\left(w^{-i}, l^{-i}\right)\right)\right\}$

be the family of all appeals that appear in either the domain or range of $b^{i}($.$) . Then$ $|L|=O\left(n^{d}\right)$.

3. There exists a constant $c$ such that every appeal $l \in L$ is $c n^{d}$-limited.

Theorem A.1 Consider a second chance mechanism with an $O\left(n^{d}\right)$-limited output algorithm. Suppose that an agent has a d-bounded revision function. For every $T^{\prime}=\Omega\left(n^{2 d}\right)$, if the mechanism is $T^{\prime}$-limited, the agent has a feasibly truthful action.

Proof: Let $i$ be the agent and let $b^{i}$ be its revision function. We again use a simulation argument in order to define the appeal $l^{i}($.$) . For every vector w^{-i}$ we compute the following outputs:

1. $o_{0}=k(w)$.

2. Similarly to the the proof of Theorem 4.4, let $L=\left\{\tau_{1} \ldots \tau_{|L|}\right\}$ be the family of all appeal functions that are in the domain or the range of $b^{i}$. For all $j=1, \ldots|L|$ define $\left.o_{j}=k\left(\tau_{j}(w)\right)\right)$.

3. Define $l(w)=\arg \max _{0 \geq j \geq|L|} g\left(\left(v^{i}, w^{-i}\right), o_{j}\right)$ as the output with the maximum welfare according to $\left(v^{i}, w^{-i}\right)$ among all the outputs defined above.

Claim A.2 $l^{i}($.$) is n^{2 d}$-limited.

Proof: W.l.o.g. the running time of $k($.$) is bounded by c n^{d}$. Otherwise, we will raise the constant. According to the definitions, the appeal $l^{i}$ performs $n^{d}+1$ computations, each requiring at most $c n^{d}$ time units. Thus, the overall computation takes at most $O\left(n^{2 d}\right)$.

Claim A.3 $a^{i}=\left(v^{i}, l^{i}\right)$ is feasibly truthful.

Proof: Assume by contradiction that there exists an action vector $a^{-i}$ in $\operatorname{dom}\left(b^{i}\right)$ such that $u\left(\left(a^{i}, a^{-i}\right)<u\left(\left(b^{i}\left(a^{-i}\right), a^{-i}\right)\right.\right.$.

Consider the case when the agent chooses $b^{i}\left(a^{-i}\right)=\left(w^{i}, \tau^{i}\right)$. The mechanism takes the output $\hat{o}$ that maximizes the welfare (according to $w$ ) from the following set $S$ of outputs: 
1. $o_{0}=k(w)$.

2. $o_{j}=k\left(l_{j}(w)\right)$ for every $j \neq i$, i.e. the result of the appeals of the other agents.

3. $o_{i}=k\left(\tau^{i}(w)\right)$.

When the agent chooses $a^{i}$, the outputs are measured according to the "right" type vector $\left(v_{i}, w^{-i}\right)$. Moreover, it is taken from the following superset of the outputs in $S$ :

1. $o_{0}^{\prime}=k\left(\left(v_{i}, w^{-i}\right)\right)$ (from the definition of the mechanism).

2. $o_{j}^{\prime}=k\left(l_{j}\left(\left(v_{i}, w^{-i}\right)\right)\right)$ for every $j \neq i$, i.e., the result of the appeals of the other agents (also, from the definition of the mechanism).

3. $o_{j}^{\prime}=k(\tau(w))$ for every $\tau \in L$. Since $a^{-i}$ is in the domain of $b^{i}$, this set includes all the outputs of the form $k\left(l_{j}(w)\right)$ from the case where $i$ chooses $b^{i}\left(a^{-i}\right)$. It also contains the result of its own appeal $\tau^{i}(w)$.

4. $k(w)$ (from the definition of $\left.l^{i}().\right)$.

Let $\tilde{o}$ be the chosen output in this case. Since the set of outputs in the second case is a superset of the first, $g\left(\left(v_{i}, w^{-i}\right), \tilde{o}\right) \geq g\left(\left(v_{i}, w^{-i}\right), \hat{o}\right)$. According to Lemma 4.2 the utility of the agent when choosing $a^{i}$ is thus higher than when choosing $b^{i}\left(a^{-i}\right)$ - a contradiction.

This completes the proof of Theorem A.1.

As in the case of appeal-independent functions, the theorem gives a prescription for constructing an appeal that guarantees the agent a feasibly dominant action.

\section{References}

Anderson, E., Kelly, F., \& Steinberg, R. (2002). A contract and balancing mechanism for sharing capacity in a communication network.. To appear.

Archer, A., \& Tardos, E. (2002). Frugal path mechanisms. In Proceedings of the 13th Annual ACM-SIAM Symposium on Discrete Algorithms, 991-999.

Banks, J., Ledyard, J., \& Porter, D. (1989). Allocating uncertain and unresponsive resources: An experimental approach. RAND Journal of Economics, 20, 1-25.

Bartal, Y., Gonen, R., \& Nisan, N. (2003). Incentive compatible multi unit combinatorial auctions. In Proceedings of Ninth Conference of Theoretical Aspects of Rationality and Knowledge, pp. 72-87.

Bartholdi, J. J., Tovey, C. A., \& Trick., M. A. (1992). How hard is it to control an election?. Mathematical and Computer Modelling (Special Issue on Formal Theories of Politics), 16, 27-40.

Carroll, T. E., \& Grosu, D. (2005). Distributed algorithmic mechanism design for scheduling on unrelated machines. In Proceedings of the 8th International Symposium on Parallel Architectures, Algorithms, and Networks, pp. 194-199. 
Clarke, E. H. (1971). Multipart pricing of public goods. Public Choice, 17-33.

Cramton, P. (1997). The fcc spectrum auction: an early assessment. Journal of Economics and Management Strategy, 431-495.

Cramton, P., Shoham, Y., \& Steinberg, R. (2006). Combinatorial Auctions. MIT Press.

d'Aspremont, C., \& Gerard-Varet, L. (1979). Incentives and incomplete information. Journal of Public Economics, 11(1), 25-45.

Elkind, E., Sahai, A., \& Steiglitz, K. (2004). Frugality in path auctions. In Proceedings of the 15th Annual ACM-SIAM Symposium on Discrete Algorithms, pp. 701-709.

Feigenbaum, J., Papadimitriou, C., \& Shenker, S. (2000). Sharing the cost of multicast transmissions. In Proceeding of the Thirty-Second Annual ACM Symposium on Theory of Computing.

Groves, T. (1973). Incentives in teams. Econometrica, 41, 617-631.

Holzman, R., Kfir-Dahav, N., Monderer, D., \& Tennenholtz, M. (2004). Bundling equilibrium in combinatorial auctions. Games and Economic Behavior, 47, 104-123.

Holzman, R., \& Monderer, D. (2004). Characterization of ex post equilibrium in the vcg combinatorial auctions. Games and Economic Behavior, 47, 87-103.

Lavi, R., Nisan, N., \& Mualem, A. (2003). Towards a characterization of truthful combinatorial auctions. In Proceedings of the 44th Annual IEEE Symposium on Foundations of Computer Science.

Lehmann, D., O‘Callaghan, L., \& Shoham, Y. (2002). Truth revelation in rapid, approximately efficient combinatorial auctions. Journal of the ACM, 49(5), 577-602. A preliminay version appeared at Proc. of the first ACM Conference on Electronic Commerce.

Mas-Collel, A., Whinston, W., \& Green, J. (1995). Microeconomic Theory. Oxford university press.

Nisan, N. (2000). Bidding and allocation in combinatorial auctions. In Proceedings of the Second ACM Conference on Electronic Commerce, pp. 1-12.

Nisan, N., \& Ronen, A. (2001). Algorithmic mechanism design. Games and Economic Behaviour, 35, 166-196. Extended abstract appeared in Proceedings of the Thirty First Annual ACM symposium on Theory of Computing.

Osborne, M. J., \& Rubinstein, A. (1994). A Course in Game Theory. MIT press.

Parkes, D. (1999). ibundle: An efficient ascending price bundle auction.. In Proceedings of the ACM Conference on Electronic Commerce (EC-99), pp. 148-157.

Porter, R., Ronen, A., Shoham, Y., \& Tennenholtz, M. (2002). Mechanism design with execution uncertainty. In In Proceedings of the 18th Conference on Uncertainty in Artificial Intelligence, pp. 414-421.

Roberts, K. (1979). The characterization of implementable choise rules. In Laffont, J.-J. (Ed.), Aggregation and Revelation of Preferences, pp. 321-349. North-Holland. Papers presented at the first European Summer Workshop of the Econometric Society. 
Ronen, A. (2001). Mechanism design with incomplete languages. In Proceedings of the Third ACM Conference on Electronic Commerce, 105-114.

Rosenschein, J. S., \& Zlotkin, G. (1994). Rules of Encounter: Designing Conventions for Automated Negotiation Among Computers. MIT Press.

Shoham, Y., \& Tanaka, K. (1997). A dynamic theory of incentives in multi-agent systems (preliminary report). In Proceedings of the Fifteenth International Joint Conferences on Artificial Intelligence, pp. 626-631.

Shoham, Y., \& Tennenholtz, M. (2001). The fair imposition of tasks in multi-agent systems. In Proceedings of the International Conference on Artificial Intelligence, pp. 10831088.

Vickrey, W. (1961). Counterspeculation, auctions and competitive sealed tenders. Journal of Finance, 8-37.

Wellman, M., Wurman, P., Walsh, W., \& MacKie-Mason, J. (2001). Auction protocols for decentralized scheduling. Games and Economic Behavior, 35, 271-303.

Zuckerman, D. (2006). Linear degree extractors and the inapproximability of max clique and chromatic number. In Proceedings of the 38th ACM Symposium on Theory of Computing, Seattle, Washington, USA. 